\title{
Driving Low-Power Wearable Systems with an Adaptively-Controlled Foot-Strike Scavenging Platform
}

\author{
Vishwa Goudar $^{1}$, Zhi Ren ${ }^{2}$, Paul Brochu ${ }^{2}$, Miodrag Potkonjak ${ }^{1}$ and Qibing Pei ${ }^{2}$ \\ ${ }^{1}$ Computer Science Department, University of California, Los Angeles \\ ${ }^{2}$ Department of Materials Science and Engineering, University of California, Los Angeles \\ vishwa@cs.ucla.edu,renz07@ucla.edu,pbrochu@ucla.edu,miodrag@cs.ucla.edu, qpei@seas.ucla.edu
}

\begin{abstract}
We explore the use of Dielectric Elastomer (DE) microgenerators as a means to scavenge energy from foot-strikes and power wearable systems. While they exhibit large energy densities, DEs must be closely controlled to maximize the energy they transduce. Towards this end, we propose a DE micro-generator array configuration that enhances transduction efficiency, and the use of foot pressure sensors to realize accurate control of the individual DEs. Statistical techniques are applied to customize performance for a user's gait and enable energy-optimized adaptive online control of the system. Simulations based on experimentally collected foot pressure datasets, empirical characterization of DE mechanical behavior and a detailed model of DE electrical behavior show that the proposed system can achieve between 45 and $66 \mathrm{~mJ}$ per stride.
\end{abstract}

\section{Author Keywords}

Energy Harvesting; Dielectric Elastomer Generators; Adaptive Control

\section{ACM Classification Keywords}

C.3 Special-Purpose And Application-Based Systems: Process Control Systems

\section{INTRODUCTION}

We focus on the need to drive low-power wearable systems with an ambient source of energy in mobile contexts. The human body offers a myriad of sources to parasitically scavenge energy with minimal discomfort. For example, foot strikes of a 154-pound person can produce upwards of 7W [4]. The harvesting system must also be comfortable to the human user and adjust to variations in her/his context. The authors of [4] review several systems for parasitic harvesting of human energy output including foot-strike energy. These span prototypes with outputs ranging from a few milli-watts to several hundred milli-watts per foot-strike.

Recently, a new class of transducers called Dielectric Elastomers (DEs) have been introduced as micro-generators.

Permission to make digital or hard copies of all or part of this work for personal or classroom use is granted without fee provided that copies are not made or distributed for profit or commercial advantage and that copies bear this notice and the full citation on the first page. Copyrights for components of this work owned by others than ACM must be honored. Abstracting with credit is permitted. To copy otherwise, or republish, to post on servers or to redistribute to lists, requires prior specific permission and/or a fee. Request permissions from permissions@ acm.org.

ISWC'13, September 9-12, 2013, Zurich, Switzerland.

Copyright (c) 2013 ACM 978-1-4503-2127-3/13/09...\$15.00.

http://dx.doi.org/10.1145/2493988.2494340

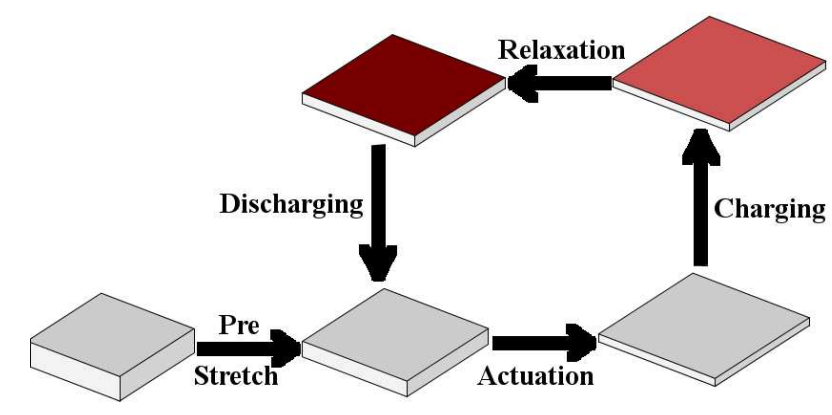

Figure 1: Dielectric elastomer generator transduction cycle

These are high energy-density rubber-like materials that can yield 5 to 40 times the energy density of piezoelectrics, making them more productive in terms of their energy output while requiring a smaller volume and mass of transducer to produce a given amount of energy. While competing technologies transduce ambient energy by exploiting underlying physical phenomena (e.g. seebeck, photovoltaic and piezoelectric effects), DEs transduce mechanical energy based on an electrostatic principle. As a result, a DE's output is heavily reliant on its control, i.e. the timing of charge during its transduction cycle. Further, in harvesting energy from foot-strikes, inter-step variability in the spatio-temporal foot pressure profile will contribute to variations in the optimum charge timing of a DE. To maximize the net energy harvested from foot-strikes with DEs, we propose (i) an array of DE microgenerators spread across the shoe sole to improves transduction efficiency, and, (ii) a power-efficient and adaptive control algorithm that enlists plantar pressure sensors to enhance the system's ability to predict optimal charge timing at each DE, as constituted by the user's gait.

HARVESTER SYSTEM CONFIGURATION AND CONTROL When operating as a generator, electrical charge must be added to an elastomer's surface when it is stretched (Figure 1). On release of the applied mechanical pressure, the elastic forces in the DE relax and are converted into electrostatic force. This may be conceptualized as an increase in electrical energy in the elastomer when it relaxes, as like charges on the same surface are brought close together and opposite charges are drawn apart on the opposing surfaces. An incompressible material, DEs exhibits change in both their area and thickness when stretched. This yields an increase in capacitance (and hence an energy gain) that is proportional to the forth power of the stretch induced in it. The larger the stretch when charge is applied, the larger 


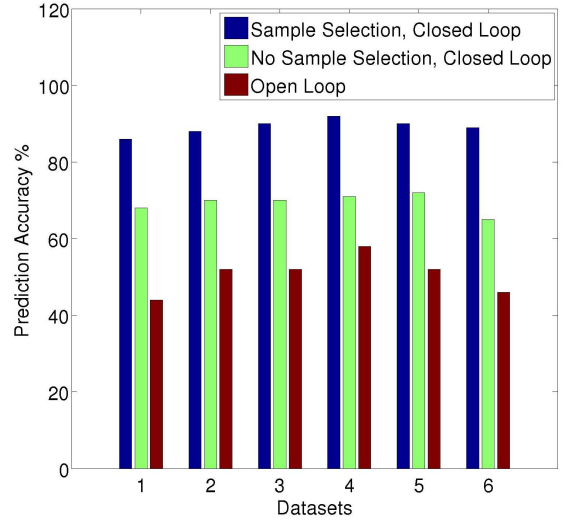

Figure 2: Prediction accuracy of different charge timing control techniques over the testing subsets

the amount of mechanical energy transduced. A shoe soleembedded DE generator will experience maximum stretch when the plantar pressure at its location is the highest, with the instant of maximum pressure depending on the location over the sole. Therefore, we propose an array of 99 shoe soleembedded DE micro-generators to harvest the mechanical energy from foot-strikes, wherein DE charge timing is individually controlled and will vary with location. We estimated the net electrical energy output of the DE micro-generators via an adaptation of the detailed transduction model in [2]. This validated model analytically derives the energy output by characterizing DE behavior in terms of the balance between the elastic and electrostatic forces acting on it. The net energy output is computed by accounting for energy consumed in charging the DE, and conduction and dielectric losses in the material. The hyper-elastic mechanical behavior of the DE was modeled with an Ogden curve, whose parameters were experimentally characterized from a prototype $\mathrm{DE}$ micro-generator.

While gait characteristics vary across people, spatial foot pressure profiles of a person will exhibit local correlation. Consequently, a few plantar pressure sensors may be used to predict the optimal charge timing of all harvesters. This is achieved with cox regression, a semi-parametric regression technqiue commonly used in time-to-event prediction. It estimates the hazard rate, or the probability that the optimal charge timing is at some instant $t$ assuming it hasn't yet occurred, based on the available plantar pressure sample values. At each instant/epoch, the hazard rate is estimated for each uncharged DE from the samples available thus far. If this indicates that the most fruitful instant for charging a DE is at the current epoch, the DE is charged. Via a step-wise regression based offline training algorithm, the location and sampling epochs of plantar pressure sensors are selected which collectively maximize the net energy harvested at all DEs. This in-

\begin{tabular}{|c|c|c|}
\hline Dataset & Dataset Description & Net Energy Output (mJ) \\
\hline 1,2 & User 1: Left, Right & $48.6,47.5$ \\
3,4 & User 2: Left, Right & $45.6,46.2$ \\
5,6 & User 3: Left, Right & $62.5,66.3$ \\
\hline
\end{tabular}

Table 1: Foot Strike Energy Harvesting Output. volves considering: (i) The energy expended in collecting the sample; (ii) The improvement in aggregate energy harvested that is attributable to the sample, which includes a goodnessof-fit test to prevent overfitting; (iii) Sampling power constraints that limit the number of sensors sampled at a time, a consequence of the instantaneous power draw of the target platform and the power rating of the battery driving it.

\section{VALIDATION}

By simulation, we validated these technqiues for the Hermes shoe - a human balance monitoring system towards gait stability analysis [3]. Hermes measures foot plantar pressure via a multi-sensory array of pressure sensors and requires $72 \mathrm{~mJ}$ per stride. Recently, a semantic accuracy preserving sampling strategy was proposed that reduces this to $43 \mathrm{~mJ}$ per stride under power constraints of at most 5 sensors sampled at a time [1]. Under these constraints, the validation was performed with plantar pressure datasets of a few hundred footsteps each, collected by Hermes at each DE location in the array. $80 \%$ of each dataset was utilized for offline training and the rest for evaluation. Table 1 lists the average energy output per stride over each dataset. Figure 2 evaluates the training algorithm by comparing the net energy output as a percentage of the output with perfect prediction accuracy for: (i) our proposed technique, (ii) one where all available samples are used in the cox-regression algorithm, and, (iii) one that estimates optimal charge timing of each harvester as its median over the training set. The second technique performs poorly due to overfitting and the third does so due to its inability to adapt.

In this work, we have evaluated the proposed design and control techniques via empirical datasets and detailed models of mechanical and electrical DE behavior. But a few factors could hinder long-term perforamance of a physically realized system. Visco-elastic losses may alter DE properties in the long term. The local correlations in plantar pressure leveraged in our closed-loop control algorithm may also change over time. Finally, losses due to inefficiencies in the rectification circuitry have not been considered while estimating energy output. The design and efficiency of this circuitry and the extent of impact of long-term performance factors will be studied in the future.

\section{REFERENCES}

1. Goudar, V., and Potkonjak, M. Power constrained sensor sample selection for improved form factor and lifetime in localized bans. In Proc. Wireless Health 2012, ACM Press (2012), Article 7.

2. Jean-Mistral, C., Basrour, S., and Chaillout, J.-J. Modelling of dielectric polymers for energy scavenging applications. Smart Materials and Structures 19, 10 (2010), Article ID 105006.

3. Noshadi, H., Ahmadian, S., Hagopian, H., et al. HERMES - mobile balance and instability assessment system. In Proc. BIOSIGNALS, INSTICC Press (2010), 264-270.

4. Paradiso, J. A., and Starner, T. Energy scavenging for mobile and wireless electronics. IEEE Pervasive Computing 4, 1 (2005), 18-27. 